\title{
Speckle interferometry of nearby multiple stars ${ }^{\star, \star \star}$
}

\author{
I. I. Balega ${ }^{1}$, Y. Y. Balega ${ }^{1}$, K.-H. Hofmann ${ }^{2}$, A. F. Maksimov ${ }^{1}$, E. A. Pluzhnik ${ }^{1,3}$, D. Schertl ${ }^{2}$, \\ Z. U. Shkhagosheva ${ }^{1,3}$, and G. Weigelt ${ }^{2}$ \\ 1 Special Astrophysical Observatory, Nizhnij Arkhyz, Zelenchuk region, Karachai-Cherkesia 369167, Russia \\ 2 Max-Planck-Institut für Radioastronomie, Auf dem Hügel 69, 53121 Bonn, Germany \\ 3 Isaac Newton Institute of Chile, SAO RAS Branch, Russia
}

Received 24 August 2001 / Accepted 18 December 2001

\begin{abstract}
We present the results of diffraction-limited optical speckle interferometry and infrared bispectrum speckle interferometry of 111 double and 10 triple systems performed in 1998-1999 with the 6-m telescope of the Special Astrophysical Observatory in Zelenchuk. The observations concentrated on nearby close binaries discovered during the Hipparcos mission. Many nearby fast-orbiting low-mass binaries known before Hipparcos were also included in the program. New companions were first resolved in 4 systems: HIP 5245, ADS 3179, Kui 99, and ADS 16138. In addition to accurate relative positions, magnitude differences were measured for most of the pairs. We combined our results with the Hipparcos parallaxes to derive absolute magnitudes and spectral types for 63 binaries and 4 triples. Preliminary orbital elements and the mass-sum are derived for HIP 689, and improved orbits are presented for HIP 16602 (CHR 117) and HIP 21280 (CHR 17).
\end{abstract}

Key words. stars: binaries: visual - stars: fundamental parameters - techniques: interferometric

\section{Introduction}

New binaries detected by Hipparcos (ESA 1997) are important targets for speckle interferometric studies. For nearby fast-moving pairs, ground-based interferometric measurements can provide reliable orbital solutions within a few years. Together with the Hipparcos parallaxes, the orbits allow the estimation of mass-sums of the components. Accurate magnitude differences can yield useful information about their luminosities and spectral types.

In this paper, we describe the first results of our speckle observations of 48 Hipparcos binaries with parallaxes $\pi_{H p}>10$ mas and separations less than $\sim 1^{\prime \prime}$ together with 73 fast-orbiting binary and multiple stars known before the Hipparcos mission from speckle surveys.

\section{Observations and data reduction}

The observations were carried out at the 6-m BTA telescope of the Special Astrophysical Observatory. The detec-

Send offprint requests to: Y. Y. Balega,

e-mail: balega@sao.ru

* Based on data collected at the Special Astrophysical Observatory, Russia

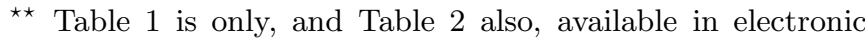
form at the CDS via anonymons ftp to cdsarc.u-strasbg.fr (130.79.128.5) or via

http://cdsweb.u-strasbg.fr/cgi-bin/qcat?J/A+A/385/87 tor of our visible speckle camera is an intensified Peltiercooled $1280 \times 1024$ pixel CCD with a multialkali S25 intensifier photocathode. The maximum frame rate of the camera is 6 speckle interferograms per second. A microscope provides a scale of 6 mas per pixel. For speckle observations we used filters with the following centre wavelength/bandwidth: $545 \mathrm{~nm} / 30 \mathrm{~nm}$ (centre of the $V$-band), $610 \mathrm{~nm} / 20 \mathrm{~nm}(G), 800 \mathrm{~nm} / 60 \mathrm{~nm}$ (centre of the $R$-band). In the following sections these filters are called as $V^{\prime}, G^{\prime}$ and $R^{\prime}$ filter. Risley prisms were used for the compensation of atmospheric dispersion. Under good seeing conditions our detector allows the observations of binaries as faint as 15 th magnitude. With the $6-\mathrm{m}$ telescope we were able to resolve stellar companions as close as 20 mas.

The speckle interferograms were taken on 710 October 1998. The image motion-compensated seeing (FWHM) was 1".0-1".5. Typically 1000 to 2000 speckle frames per object were taken with exposure times from 5 to $20 \mathrm{~ms}$. The instrumental scale calibration was performed by recording interference fringes from a two-hole mask installed in a converging beam between the primary mirror and the focal plane; the projection of the distance between the holes on the mirror surface was $4968 \mathrm{~mm}$.

In our optical speckle interferometry (Labeyrie 1970) observations the relative position and magnitude difference of each binary was derived from the ensemble average of all power spectra of speckle interferograms without compensation of the atmospheric transfer function. 
However, in the case when a close binary at the diffraction limit of the telescope was expected, we observed a reference star for the compensation.

To determine the magnitude difference, we measured the value $A^{2}+B^{2} / A B$, where $A$ and $B$ are the relative intensities of the components, as a function of the spatial frequency for each circle around the center of the ensemble average power spectrum up to the diffraction cut-off frequency. The $A^{2}+B^{2} / A B$ value represents the fringe contrast in the spectrum. The contrast of each circle intensity distribution depends on both the flux ratio $A / B$ of the binary and the photon bias (Goodman \& Belsher 1977). For bright binaries, the photon bias is weak and has small influence on the contrast of the fringes in the power spectrum. Therefore, in the case of bright pairs, the contrast is independent of the radius of the circles and the intensity distribution on each circle yields approximately the same value for the magnitude difference. However, for faint objects, the additive photon bias function depends on the power spectrum circle radius (the photon bias decreases slowly from the centre to higher spatial frequences) as the photoevents are extended dots with a diameter of approximately $3 \times 3$ pixel. In this case the radius-dependent fringe contrast measurements are used to fit both the shape of the photon bias function and the magnitude difference of the binary. This method can only be applied if the power spectrum consists of more than 3 fringes. The $180^{\circ}$ position angle ambiguity of the optical speckle interferometry observations was overcome by the Walker method (1981).

The infrared speckle observations of 21 stars were performed on 27-29 September 1999. The seeing was 1". $0-1$ ". 7 . The speckle interferograms were recorded through a $K^{\prime}$-band filter with a centre wavelength/bandwidth of $2115 \mathrm{~nm} / 214 \mathrm{~nm}$. The detector used for the observations was a HAWAII camera (frame rate 3 frames/s for $256^{2}$ subframes). The pixel size was 26.4 mas and the field of view was $6 . \prime 8$. For each object, 300 to 700 speckle interferograms were recorded with exposure times between 10 and 240 msec. The diffraction-limited infrared images were reconstructed using the bispectrum speckle interferometry method (Weigelt 1977; Lohmann et al. 1983; Hofmann et al. 1995). $K^{\prime}$-band images with diffraction-limited resolution of 76 mas were obtained.

\section{Results and discussion}

\subsection{Binary star measurements}

In Table 1, our 162 speckle measurements of 111 pairs are presented together with the results of differential photometry for most of the systems. The first three columns in Table 1 list the Hipparcos number (ESA 1997), the name of the star or its catalog number, and the discoverer designation. Column four contains the epoch-2000 coordinates. Column five gives the epoch of observation, shown as the fractional Besselian year. The next four columns list the measured position angle $\theta^{\circ}$ measured in degrees for the secondary relative to the primary, angular distance $\rho$ in mas, and their errors. They are followed by the measured magnitude difference together with its error. Finally, the last column contains the filter indication. The note "UR" stands for unresolved stars. Comments to some of the measurements are given in Sect. 3.5.

The formal errors of relative position measurements, obtained from least-squares fitting, are $0.3-0.5^{\circ}$ and 1-2 mas for $\theta$ and $\rho$ measurements, respectively. For wellstudied binaries, observed as standard stars for speckle interferometry, the root mean square deviation of residuals of our measures to the ephemeris was found to be $0.5^{\circ}$ in position angle and 2.5 mas in separation. For 27 binary systems the $180^{\circ}$ position angle ambiguity was not resolved. These cases are marked with an asterisk in Table 1.

\subsection{Triple systems}

The triple star measurements are presented in Table 2. The column headings are similar to Table 1, with the additional column for the subsystem identification. For all triples, position angles are given with vectors starting at the position of the brightest component. In three cases the third component of the system was directly resolved for the first time: a distant dwarf in the system HIP 5245, close sub-systems for spectroscopic binaries HIP 101955 (Kui 99A), and HIP 111805 (ADS 16138A).

\subsection{Magnitude differences}

Tables 1 and 2 give the magnitude differences for 93 binaries and 9 triples. For the triple systems $\Delta m$ 's were computed in the similar way as for the binaries, i.e. by measurements of the fringe contrast in the power spectrum versus spatial frequency. Unfortunately, intereference filters for speckle interferometry (e.g., $V^{\prime}: 545 \mathrm{~nm} / 30 \mathrm{~nm}$ ) are considerably narrower than the standard Johnson system or the Hipparcos photometric band (passband maximum/ bandwidth: $450 \mathrm{~nm} / 220 \mathrm{~nm}$ ). Therefore the direct comparison of our $\Delta m$ 's with the Hipparcos magnitude differences $\Delta H p$ is difficult. In Fig. 1 we compare our $V^{\prime}$ magnitude differences with the Hipparcos $\Delta H p$ as our $V^{\prime}$ band is close to the center of the broad-band photometric system of the astrometric satellite. The leastsquares approximation of the relation between $\Delta m_{\mathrm{BTA}}$ in the $V^{\prime}$ filter and $\Delta H p$ yields

$\Delta m_{\mathrm{BTA}}=0.94 \Delta H p+0.13$

For this comparison we only plotted pairs with a separation of $0^{\prime \prime} 2<\rho<0$.' 5 as Hipparcos measurements of pairs with $\rho<0$ ". 2 may be inaccurate, while speckle observations of pairs with $\rho>0$. 5 may be systematically biased due to the cutting of image wings by the detector window. All $\Delta m_{\mathrm{BTA}}$ values for pairs with $\rho>0, .5$ should be used with care (marked with an asterisk in Table 1). 
Table 2. Triple star measurements.

\begin{tabular}{|c|c|c|c|c|c|c|c|c|c|c|c|c|}
\hline$\overline{\mathrm{HIP}}$ & $\mathrm{ADS}$ & Disc. & Comp. & $\begin{array}{c}\text { Coord. } \\
2000\end{array}$ & $\begin{array}{c}\text { Epoch } \\
1990.0+\end{array}$ & $\theta^{\circ}$ & $\sigma_{\theta}$ & $\begin{array}{l}\rho \\
\text { mas }\end{array}$ & $\begin{array}{l}\sigma_{\rho} \\
\operatorname{mas}\end{array}$ & $\Delta m$ & $\sigma_{\Delta m}$ & Filter \\
\hline \multirow[t]{4}{*}{4440} & ADS 784 & BU 1099 & $\mathrm{AB}$ & $00565+6022$ & 8.7744 & 349.0 & 0.6 & 286 & 3 & & & $V^{\prime}$ \\
\hline & & & & & 8.7773 & 347.8 & 0.4 & 291 & 2 & & & $V^{\prime}$ \\
\hline & & BAG 10 & AP & & 8.7744 & 98.3 & 13.6 & 20 & 5 & & & $V^{\prime}$ \\
\hline & & & & & 8.7773 & 69.8 & 14.3 & 16 & 5 & & & $V^{\prime}$ \\
\hline \multirow[t]{3}{*}{5245} & & & $\mathrm{AB}$ & 01071-0036 & 8.7745 & 269.5 & 0.5 & 275 & 2 & 1.61 & 0.11 & $R^{\prime}$ \\
\hline & & & & & 9.7473 & 262.7 & 1.5 & 263 & 4 & 1.12 & 0.25 & $K^{\prime}$ \\
\hline & & & $\mathrm{AC}$ & & 9.7473 & 173.3 & 1.0 & 1122 & 5 & 2.97 & 0.12 & $K^{\prime}$ \\
\hline \multirow[t]{2}{*}{21730} & ADS 3358 & BU 1295 & $\mathrm{AB}$ & $04400+5328$ & 8.7775 & 154.2 & 0.5 & 278 & 2 & 0.89 & 0.03 & $G^{\prime}$ \\
\hline & & STF 566 & $\mathrm{AC}$ & & 8.7775 & 194.8 & 0.5 & 780 & 2 & 1.55 & 0.03 & $G^{\prime}$ \\
\hline \multirow[t]{2}{*}{84934} & HR 6466 & KUI 80 & & $17215+2845$ & 8.7766 & 166.9 & 0.5 & 700 & 2 & 2.41 & 0.07 & $V^{\prime}$ \\
\hline & & CHR 194 & & & 8.7766 & 130.8 & 2.84 & 45 & 3 & 0.50 & 0.07 & $V^{\prime}$ \\
\hline \multirow[t]{2}{*}{90284} & ADS 11344 & HU 66 & $\mathrm{AB}$ & $18253+4846$ & 8.7795 & 236.6 & 0.5 & 269 & 2 & 0.70 & 0.02 & $G^{\prime}$ \\
\hline & & STT 351 & $\mathrm{AC}$ & & 8.7795 & 21.4 & 0.5 & 726 & 2 & 0.11 & 0.02 & $G^{\prime}$ \\
\hline \multirow[t]{2}{*}{101955} & & KUI 99 & $\mathrm{AB}$ & $20396+0458$ & 8.7741 & 124.4 & 0.5 & 377 & 2 & 0.88 & 0.05 & $G^{\prime}$ \\
\hline & & & $\mathrm{AC}$ & & 8.7741 & 55.0 & 0.9 & 161 & 3 & 1.09 & 0.05 & $G^{\prime}$ \\
\hline \multirow[t]{2}{*}{104642} & ADS 14749 & STF 2780 & $\mathrm{AB}$ & $21118+5959$ & 8.7770 & 213.8 & 0.5 & 1032 & 2 & & & $V^{\prime}$ \\
\hline & & MCA 67 & $\mathrm{AC}$ & & 8.7770 & 157.6 & 5.6 & 26 & 3 & 0.23 & 0.07 & $V^{\prime}$ \\
\hline \multirow[t]{2}{*}{111805} & ADS 16138 & HO 295 & $\mathrm{AB}$ & $22388+4419$ & 8.7743 & 330.4 & 0.5 & 157 & 2 & 0.18 & 0.07 & $V^{\prime}$ \\
\hline & & & $\mathrm{AC}$ & & 8.7743 & 357.7 & 11.4 & 18 & 4 & 1.27 & 0.07 & $V^{\prime}$ \\
\hline \multirow[t]{2}{*}{112170} & ADS 16214 & STT 476 & $\mathrm{AB}$ & $22431+4710$ & 8.7771 & 301.5 & 0.5 & 493 & 2 & 0.59 & 0.04 & $G^{\prime}$ \\
\hline & & HU 91 & $\mathrm{BC}$ & & 8.7771 & 33.0 & 2.7 & 85 & 4 & 0.82 & 0.02 & $G^{\prime}$ \\
\hline \multirow[t]{2}{*}{116726} & ADS 16904 & A 643 & $\mathrm{BA}$ & $23393+4543$ & 8.7745 & 333.3 & 0.5 & 237 & 2 & 0.13 & 0.04 & $V^{\prime}$ \\
\hline & & CHR 149 & $\mathrm{AC}$ & & 8.7745 & 223.1 & 2.0 & 29 & 2 & 0.96 & 0.03 & $V^{\prime}$ \\
\hline
\end{tabular}

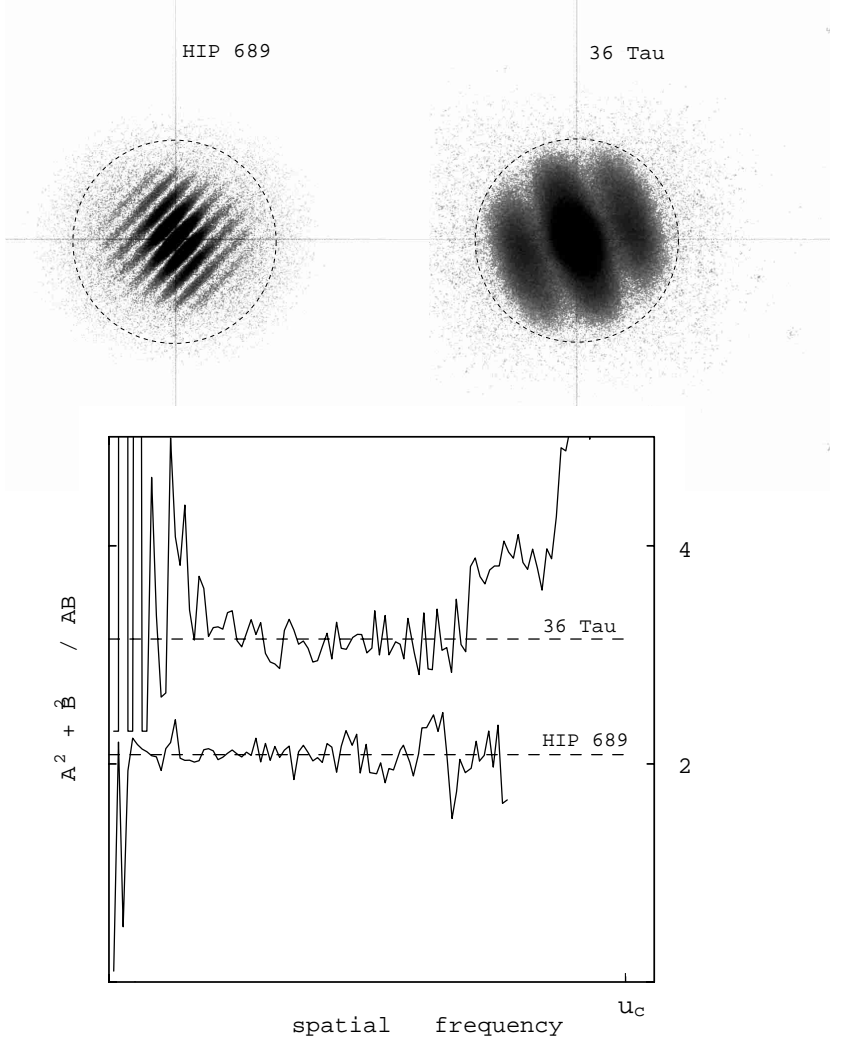

Fig. 1. Top: Ensemble average power spectra for HIP 689 and 36 Tau showing the fringes corresponding to the separations 133 mas and 32 mas. Dotted circles represent the diffraction cut-off frequency at $565 \mathrm{~nm}$. Bottom: Corresponding $A^{2}+B^{2} / A B$ value as a function of the spatial frequency. The cut-off limit $u_{\mathrm{c}}$ is indicated.

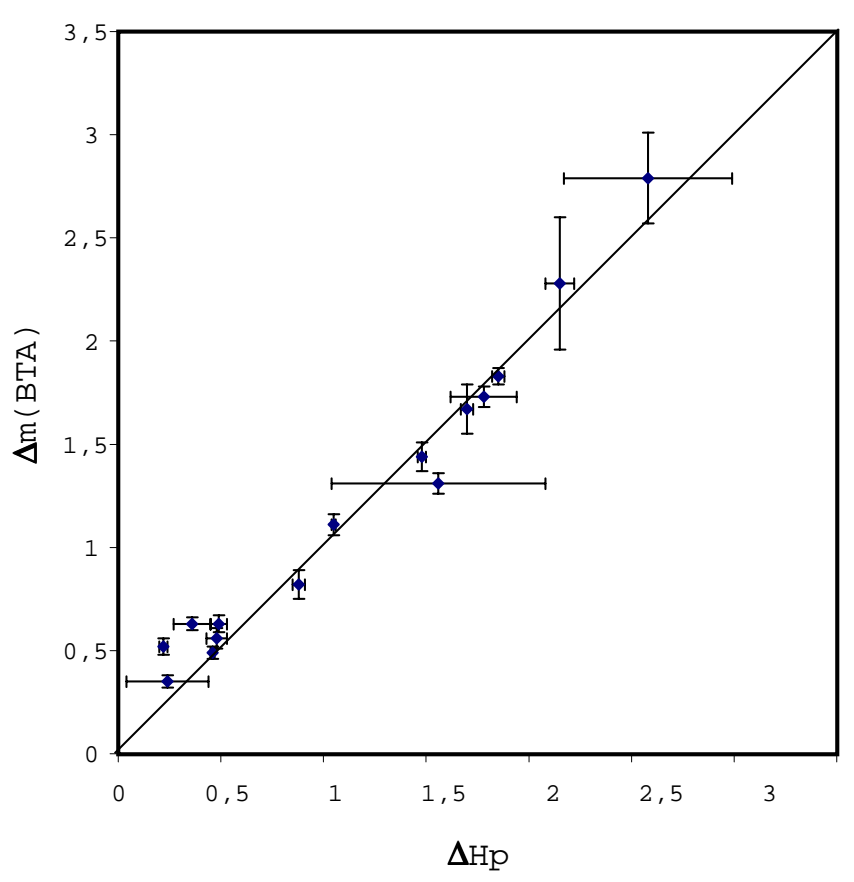

Fig. 2. Comparison of Hipparcos magnitude differences with the speckle measurements made in the $V^{\prime}(545 \mathrm{~nm} / 30 \mathrm{~nm})$ filter at the BTA telescope.

\subsection{Absolute magnitudes and spectral types}

Our magnitude differences can be combined with total $V$ magnitudes and Hipparcos parallaxes in order to obtain absolute magnitudes and spectral types of the individual components. Because of the difference between 
Table 3. Absolute magnitudes and spectral types of binary star components.

\begin{tabular}{|c|c|c|c|c|c|c|c|c|c|c|c|}
\hline HIP & $M_{\mathrm{A}}$ & $M_{\mathrm{B}}$ & Filter & $S p_{\mathrm{A}}$ & $S p_{\mathrm{B}}$ & HIP & $M_{\mathrm{A}}$ & $M_{\mathrm{B}}$ & Filter & $S p_{\mathrm{A}}$ & $S p_{\mathrm{B}}$ \\
\hline 461 & 3.4 & 4.7 & $V^{\prime}$ & F5 & G2 & 20895 & 9.1 & 9.4 & $R^{\prime}$ & M1 & M1 \\
\hline 689 & 3.5 & 3.8 & $V^{\prime}$ & F5 & F8 & 21280 & 5.7 & 7.0 & $G^{\prime}$ & G9 & K4 \\
\hline 768 & 3.9 & 5.5 & $V^{\prime}$ & F8 & G8 & 22550 & 3.9 & 4.2 & $V^{\prime}$ & F8 & F9 \\
\hline 1055 & 7.9 & 9.1 & $R^{\prime}$ & $\mathrm{K} 7$ & M0 & 23317 & 7.1 & 8.6 & $R^{\prime}$ & $\mathrm{K} 4$ & M0 \\
\hline 1392 & 3.4 & 4.5 & $V^{\prime}$ & F5 & G0 & 23418 & 9.3 & 10.3 & $R^{\prime}$ & M1 & M3 \\
\hline 3361 & 6.5 & 7.6 & $R^{\prime}$ & $\mathrm{K} 2$ & K6 & 25240 & 4.8 & 5.1 & $G^{\prime}$ & G2 & G5 \\
\hline 3795 & 4.1 & 4.8 & $V^{\prime}$ & F8 & G2 & 26926 & 2.9 & 3.8 & $V^{\prime}$ & $\mathrm{F} 1$ & F8 \\
\hline 4809 & 3.1 & 3.3 & $V^{\prime}$ & & & 27758 & 3.9 & 5.2 & $V^{\prime}$ & F8 & G5 \\
\hline 4841 & 3.3 & 4.4 & $G^{\prime}$ & F2 & G0 & 83064 & 3.2 & 3.9 & $R^{\prime}$ & F1 & F8 \\
\hline 4849 & 6.7 & 8.4 & $G^{\prime}$ & K3 & K8 & 83791 & 3.7 & 4.5 & $R^{\prime}$ & F6 & G1 \\
\hline 5531 & 3.2 & 4.0 & $V^{\prime}$ & $\mathrm{F} 2$ & F8 & 87991 & 7.5 & 7.7 & $R^{\prime}$ & K5 & K6 \\
\hline 6730 & 8.4 & 8.8 & $R^{\prime}$ & $\mathrm{K} 8$ & M0 & 88136 & 2.6 & 3.1 & $V^{\prime}$ & F0 & $\mathrm{F} 1$ \\
\hline 7338 & 8.2 & 9.7 & $R^{\prime}$ & K8 & M2 & 94056 & 7.7 & 8.1 & $R^{\prime}$ & K6 & $\mathrm{K} 7$ \\
\hline 7580 & 3.6 & 4.8 & $V^{\prime}$ & F5 & G3 & 94252 & 4.6 & 5.3 & $R^{\prime}$ & G2 & G6 \\
\hline 8258 & 4.2 & 4.4 & $V^{\prime}$ & F9 & G0 & 95995 & 6.1 & 6.4 & $V^{\prime}$ & $\mathrm{K} 1$ & $\mathrm{~K} 2$ \\
\hline 11253 & 4.4 & 7.2 & $V^{\prime}$ & G0 & $\mathrm{K} 4$ & 96656 & 6.2 & 6.8 & $V^{\prime}$ & K1 & K4 \\
\hline 11352 & 5.5 & 5.7 & $V^{\prime}$ & G8 & G9 & 105371 & 3.5 & 5.9 & $R^{\prime}$ & F5 & K0 \\
\hline 12446 & 7.4 & 7.8 & $R^{\prime}$ & K5 & $\mathrm{K} 7$ & 105947 & 3.9 & 5.4 & $V^{\prime}$ & F8 & G8 \\
\hline 12986 & 7.0 & 8.1 & $R^{\prime}$ & K4 & $\mathrm{K} 7$ & 106394 & 5.8 & 7.8 & $R^{\prime}$ & K0 & K6 \\
\hline 13117 & 9.4 & 10.4 & $R^{\prime}$ & M1 & M3 & 106439 & 6.8 & 7.9 & $R^{\prime}$ & $\mathrm{K} 4$ & K7 \\
\hline 14075 & 5.5 & 5.5 & $R^{\prime}$ & G8 & G8 & 106972 & 9.9 & 11.1 & $R^{\prime}$ & M2 & M4 \\
\hline 14230 & 4.7 & 6.4 & $V^{\prime}$ & $\mathrm{G} 2$ & $\mathrm{~K} 2$ & 107181 & 7.8 & 9.0 & $R^{\prime}$ & K6 & M0 \\
\hline 14524 & 7.6 & 8.0 & $R^{\prime}$ & K6 & K7 & 109281 & 2.6 & 3.1 & $R^{\prime}$ & & \\
\hline 14669 & 9.5 & 11.2 & $R^{\prime}$ & M2 & M4 & 111685 & 8.2 & 10.2 & $R^{\prime}$ & $\mathrm{K} 7$ & M3 \\
\hline 14864 & 7.6 & 9.1 & $R^{\prime}$ & K6 & M1 & 111805 & 4.5 & 5.0 & $V^{\prime}$ & G0 & G5 \\
\hline 14929 & 4.0 & 4.2 & $V^{\prime}$ & F8 & F9 & 112695 & 3.6 & 5.6 & $G^{\prime}$ & & \\
\hline 16025 & 4.1 & 5.8 & $V^{\prime}$ & F8 & K0 & 114444 & 4.4 & 6.3 & $V^{\prime}$ & G0 & K2 \\
\hline 16602 & 2.8 & 3.0 & $V^{\prime}$ & F0 & F1 & 114927 & 6.6 & 6.6 & $R^{\prime}$ & K3 & K3 \\
\hline 17491 & 5.7 & 8.4 & $V^{\prime}$ & G9 & K8 & 116259 & 4.6 & 7.2 & $V^{\prime}$ & G2 & $\mathrm{K} 4$ \\
\hline 19206 & 4.1 & 5.5 & $G^{\prime}$ & F8 & G8 & 116294 & 4.4 & 6.4 & $G^{\prime}$ & G0 & $\mathrm{K} 2$ \\
\hline 20087 & 2.1 & 4.2 & $V^{\prime}$ & $\mathrm{A}$ & G0 & 116810 & 7.2 & 9.2 & $R^{\prime}$ & $\mathrm{K} 4$ & M1 \\
\hline 20777 & 8.9 & 9.1 & $R^{\prime}$ & M0 & M1 & & & & & & \\
\hline
\end{tabular}

our narrow-band filters and the standard Johnson system, only rough classification is possible. The $\Delta m$ 's in the $K^{\prime}$-band were not used for the component classification. The results for 63 systems are presented in Table 3 . Column four in the table indicates the filter used for the $\Delta m$ measurement. Spectral types of the components were derived from the empirical $S p-M_{V}$ relation for main sequence stars (Lang 1992). The computed absolute magnitudes for 3 pairs (HIP 4809, HIP 109281, and HIP 112695) do not correspond to their $(B-V)$ or $(V-I)$ colours. HIP 4809 and HIP 109281 have evolved of the main sequence. The presense of a third body or an evolved component can be the explanation for 1.5 mag higher luminosity of HIP 112695. In these three cases we do not give the estimate of the spectral type in Table 3.

\subsection{Binary star orbits}

For three systems the orbital solutions were found using the Hipparcos data and the speckle interferometric measurements. All observations were taken to be of unit weight. The preliminary orbits were calculated by applying the Fourier transform of the equations of motion (Monet 1979). For small number of observations this method appears to offer significant advantages over traditional configuration space techniques. On the next step, differential corrections were applied to improve the solutions. The results of the analysis are presented below in notes to individual systems. The orbit plots for three pairs are shown in Fig. 3.

\subsection{Notes to individual systems}

HIP 689. The following preliminary orbit can be estimated for this system using the first Hipparcos resolution, the measurements of Horch et al. (1999), Mason et al. (2001) and our two speckle observations:

$P=19.3^{y}, T_{0}=2005.6, e=0.38, a=0^{\prime \prime} 124$,

$i=125^{\circ}, \omega=107^{\circ}, \Omega=42^{\circ}$.

To obtain the solution we had to change the quadrant for all observations except for two measurements with 


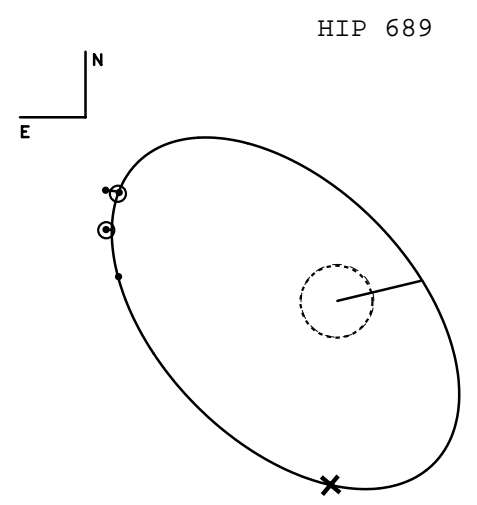

CHR 117

CHR 17
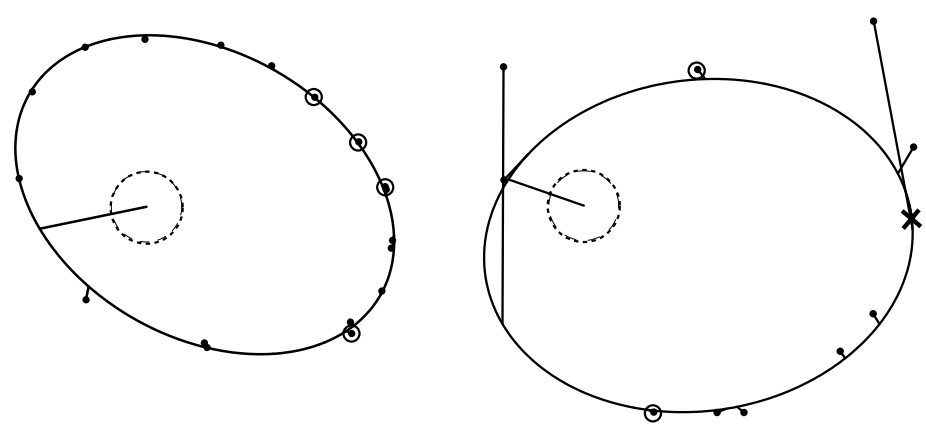

Fig. 3. Relative visual orbits for HIP 689, CHR 117 and CHR 17. BTA speckle measures (open circles), measures by other speckle observers (small filled cicles) and Hipparcos data (crosses) are shown connected to their predicted positions on the orbit, although most of the residuals are too small to show up in this manner. The periastron is indicated by a solid line. The dotted circle in the center represents the 20 mas limit of resolution. Two measures of CHR 17 with large residuals have not been taken into account for the orbital solution.

at 6-m telescope. This is explained by the following: the Hipparcos $\Delta H p=0.04$ is given with an error of $\pm 0.5 \mathrm{mag}$, while speckle observations taken with the WIYN 3.5-m telescope at Kitt Peak and the 2.1-m Struve reflector of McDonald Observatory contain $180^{\circ}$ uncertainty in position angle. Our independent image reconstructions in the visual and in the infrared give the same quadrant. The dynamical mass-sum is $2.3 M_{\odot}$.

HIP 1055. Because of the contradictory data about the total $V$ magnitude in SYMBAD and in the Hipparcos Catalogue, we used Tycho's $V_{\mathrm{T}}=10.65$ to derive the spectral types of the components.

HIP 4440. The component designations correspond to Balega et al. (1999). We could not obtain $\Delta m$ 's for this triple star because of the very small separation in the $A B$ system.

HIP 4809. A binary with evolved components. A period of 14 years is expected from the fast orbital motion of the pair.

HIP 4849 (GJ 3071). Fast orbital motion to the periastron.

HIP 5245. A new distant component was first observed in $K^{\prime}$ band. Our magnitude differences yield absolute magnitudes and spectral types $5.6, \mathrm{~K} 0 \mathrm{~V}$ and $7.2, \mathrm{~K} 4 \mathrm{~V}$ for $\mathrm{A}$ and B star, correspondingly, while the third component is probably a late $\mathrm{M}$ dwarf.

HIP 7338. Fast orbital motion with the possible periastron passage in 1999 .

HIP 7580 (Kui 7). Martin \& Mignard (1998) derived masses of $0.89 M_{\odot}$ and $1.22 M_{\odot}$ for the components using the Hipparcos parallax and $\Delta m=0.41$. We measured a significantly larger $\Delta m$, and derived the masses $1.40 M_{\odot}$ and $0.95 M_{\odot}$ for F5V and G3V components. The masssum is in agreement with the orbit of Hartkopf et al. (1996).

HIP 14075. Fast orbital motion with the possibility of periastron passage in 1999.

HIP 14230. Fast relative motion.
HIP 14669 (GJ 125). Fast relative motion.

HIP 14929. Spectral type G5 from SIMBAD do not correspond to $B-V=0.526$ and our F8-F9 spectral type.

HIP 16602 (CHR 117). Preliminary orbital solutions were given for this pair by McAlister et al. (1992) and Soederjelm (1999). With our last measure the improved orbital parameters are:

$P=14.47 \pm 0.07^{y}, T_{0}=1990.15 \pm 0.03, e=0.35 \pm 0.01$, $a=0^{\prime \prime} .120 \pm 0^{\prime \prime} 001, i=46^{\circ} \pm 1^{\circ}, \omega=236^{\circ} \pm 1^{\circ}, \Omega=$ $235^{\circ} \pm 1^{\circ}$.

Only speckle observations were used for this solution. We had to change the quadrants of all measurements obtained before 1996. The true quadrant was derived from the speckle masking image reconstructions in the infrared (Balega et al. 2001) and from our last observation in 1998.7774 .

$B-V=0.523$ corresponds to $\mathrm{F} 8$ spectral type, while $\pi_{H p}=13.9$ mas, total $V$ magnitude 6.39 and our $\Delta m$ better fit to F0 dwarf components. The orbital mass-sum of $3.06 M_{\odot}$ also corresponds to F0 dwarfs.

HIP 20531 (ADS 3179 B). This A1Vp star was observed as a reference source for ADS 3161. We resolved it for the first time as a close double with the magnitude difference close to zero.

HIP 20777 (DF Tau). Because of variable brightness, we used the median value $H p=12.08$ and obtained the spectral type M0-M1 for the components.

HIP 21280 (CHR 17). Our relative positions for this Hyades cluster binary does not match the Soederjelm's (1999) orbit: the residuals are $13.5^{\circ}$ in $\theta$ and 27 mas in $\rho$. The total mass of the system deduced from that orbit $\left(4.94 M_{\odot}\right)$ is also inconsistent with the mass-luminosity relation. A reliable orbit is achieved with the following parameters:

$P=14.05 \pm 0.24^{y}, T_{0}=1995.86 \pm 0.05, e=$ $0.61 \pm 0.02, a=0^{\prime \prime} 148 \pm 00^{\prime \prime} 005, i=45^{\circ} \pm 3^{\circ}$, $\omega=116^{\circ} \pm 6^{\circ}, \Omega=306^{\circ} \pm 6^{\circ}$. 
Speckle interferometric data and one Hipparcos observation were used to compute the orbit. Again, similar to the CHR 117 orbit, we have changed the quadrants for all measurements obtained before 1988.66 (Hartkopf et al. 2001). One measurement of Hartkopf et al. in 1990.76 and one measurement of Patience et al. in 1996.98 (Hartkopf et al. 2001) have not been taken into account because they do not fit to other data. The proper quadrant was defined from the Hipparcos measurement and our two observations. With the spectral types from Table 3 we can expect a total mass of $1.40 M_{\odot}$, while the dynamical mass-sum derived from the above orbital elements and the Hipparcos parallax is $1.23 M_{\odot}$.

HIP 83064 (Cou 1289). The derived magnitudes and spectral types of $\mathrm{F} 1+\mathrm{F} 8$ do not agree with the color $B-V=0.583$. An evolved component or a multiplicity could be an explanation.

HIP 83791 (Cou 1291). Same comment for HIP 83064.

HIP 90284 (ADS 11344). The Hipparcos parallax 5.32 mas and our $\Delta m$ 's lead to absolute magnitudes of $1.9+2.6$ ( $A B$ subsystem) and 2.0 (component $\mathrm{C})$, corresponding to spectral types $\mathrm{A} 5+\mathrm{F} 0$ and $\mathrm{A} 5$.

HIP 101955 (Kui 99). The $920^{d}$ spectroscopic subsystem of the visual pair Kui 99 is resolved for the first time. The difference $\Delta m_{V}=1.77$ between the SB2 components $\mathrm{Aa}$ and Ab, deduced by Duquennoy (1987) from the correlation dips detected by the CORAVEL spectrometer, agrees fairly well with our $\Delta m_{\mathrm{AC}}=1.09 \pm 0.05$ in the $600 \mathrm{~nm}$ filter. Using our $\Delta m$ values, $\pi_{H p}=53.8$ mas, and the total $V$ magnitude 7.88 (ESA 1997), we derive the following absolute magnitudes and spectral types for the three dwarf components of HIP 101955:

A: $7.2, \mathrm{~K} 4 \mathrm{~V}, \mathrm{~B}: 8.1, \mathrm{~K} 7 \mathrm{~V}, \mathrm{C}: 8.3, \mathrm{~K} 8 \mathrm{~V}$.

Here the components $\mathrm{A}$ and $\mathrm{C}$ correspond to the fast spectroscopic subsystem. Future observations will allow a precise determination of both orbits of the system.

HIP 105947. Further speckle observations are needed to determine the true quadrant.

HIP 106972 (GJ 4210). Fast orbital motion.

HIP 109281. The G2V spectral classification (Harlan \& Taylor 1970), used in SIMBAD, is evidently wrong. Absolute magnitudes and the mean $B-V$ color index of the components indicate the luminosity class III. Yoss (1961) also classified the star as a late-type CN giant from the objective-prism spectra at the cyanogen band at $\lambda 4216$.

HIP 111685 (GJ 4287). Fast orbital motion with a possible period of a few hundred days.

HIP 111805 (HO 295). The $551^{d}$ spectroscopic pair discovered by Duquennoy (1987) with the radial velocity scanner was resolved for the first time with the $6-\mathrm{m}$ telescope. We suggest that the spectroscopic subsystem is the more luminous component A of the pair, not B as supposed by Duquennoy from the CORAVEL data. The Aa star is brighter than the B star by $0.18 \pm 0.07$ mag. The magnitude difference between the subsystem $\mathrm{Aa}+\mathrm{Ab}$ and $\mathrm{B}$ is $0.46 \pm 0.09 \mathrm{mag}$ which is in agreement with the Hipparcos $\Delta H p=0.51 \pm 0.07 \mathrm{mag}$. From our $\Delta m$ 's,
$\pi_{H p}=26.3$ mas, and the total $V$ magnitude 6.83 , we find the following absolute magnitudes and spectral types for the components:

Aa: 4.75, G2V; Ab: 6.08, K1V; B: 4.93, G5V.

In this model there is no need to identify the less massive component $\mathrm{B}$ with the brightest one, as proposed by Duquennoy.

HIP 112695. The derived absolute magnitude of the main component $M_{\mathrm{A}}=3.6$ does not correspond to the colour index $B-V=0.683$.

HIP 114922 (GJ 893.4). A pair of M-dwarfs with fast relative motion.

\section{Summary}

Our speckle study has focused on new binaries discovered by Hipparcos and other nearby $(\pi>10$ mas) low-mass systems. The aim was to select the binaries with fast relative motion to determine new orbits and to combine the Hipparcos parallaxes with the speckle interferometric $\Delta m$ measurements to derive absolute magnitudes and spectral types of the components.

Our sample of stars included 48 new Hipparcos doubles with parallaxes above 10 mas and separations less than $\sim 1^{\prime \prime}$. The binarity was confirmed for all observed Hipparcos pairs, except HIP 15597. About 10 astrophysically interesting systems with fast orbital motion were found among the observed Hipparcos pairs.

Magnitude differences were estimated for 93 pairs and 9 triples with an error in the range between \pm 0.02 and \pm 0.50 mag. Absolute magnitudes and approximate spectral types were found for the components of 63 binary systems and for 4 triples using our speckle $\Delta m$ 's, the Hipparcos parallaxes and the known total $V$ magnitudes.

Close spectroscopic subsystems were first resolved in the hierarchical triples Kui 99A and ADS 16138. A preliminary orbit for the new F5V+F8V Hipparcos binary HIP 689 was derived from the first Hipparcos observation and the speckle data. The orbital elements of HIP 16602 (CHR 117) and the Hyades cluster binary HIP 21280 (CHR 17) were improved.

Acknowledgements. We thank the BTA telescope team for their support during the observations. We are also grateful to V. Vasyuk and A. Tokovinin for their help. This research has made use of the SIMBAD database, operated at CDS, Strasbourg, France, and the Washington Double Star Catalog of the US Naval Observatory. A support of the Russian Federal Program "Astronomy" and the Russian Foundation for Basic Research is acknowledged by YYB.

\section{References}

Balega, I. I., Balega, Y. Y., Maksimov, A. F., et al. 1999, A\&AS, 140, 287

Balega, I. I., Balega, Y. Y., Hofmann, K.-H., \& Weigelt, G. 2001, AstL, 27, 95 
Duquennoy, A. 1987, A\&A, 178, 114

ESA 1997, The Hipparcos and Tycho Catalogues, ESA SP-1200 Goodman, J. W., \& Belsher, J. F. 1977, Report TR-77-165, ARPA Order No. 2646

Harlan, E. A., \& Taylor, D. C. 1970, AJ, 75, 165

Hartkopf, W. I., Mason, B. D., \& McAlister, H. A. 1996, AJ, 111,936

Hartkopf, W. I., Mason, B. D., Wycoff, G. L., \& McAlister, H. A. 2001, Fourth Catalog of Interferometric Measurements of Binary Stars, CHARA Contrib. 4 (http://www.ad.usno.navy.mil/wds/int4.html)

Hofmann, K.-H., Seggewiss, W., \& Weigelt, G. 1995, A\&A, 300,403

Horch, E. P., Ninkov, Z., van Altena, W. F., et al. 1999, AJ, 117,548
Labeyrie, A. 1970, A\&A, 6, 85

Lang, K. R. 1992, Astrophysical Data: Planet and Stars (Springer-Verlag, NY)

Lohmann, A. W., Weigelt, G., \& Wirnitzer, B. 1983, Ap. Opt., 22,4028

Martin, C., \& Mignard, F. 1998, A\&A, 330, 585

Mason, B. D., Hartkopf, W. I., Holdenried, E. R., \& Raferty, T. J. 2001, AJ, 121, 3224

McAlister, H. A., Hartkopf, W. I., \& Mason, B. D. 1992, AJ, 104, 1961

Monet, D. G. 1979, AJ, 234, 275

Soederhjelm, S. 1999, A\&A, 341, 121

Walker, J. G. 1981, Opt. Acta, 28, 735

Weigelt, G. 1977, Opt. Commun., 21, 55

Yoss, K. N. 1961, ApJ, 134, 809 